\section{Spectral Characteristics of the Near-Wall Turbulence in an Unsteady Channel Flow}

\section{Sedat F. Tardu}

Laboratoire des Ecoulements Géophysiques et Industriels, B.P. 53 X, 38041 Grenoble, France

e-mail: sedat.tardu@hmg.inpg.fr

The modulation characteristics of the turbulent wall shear stress and longitudinal intensities in the inner layer are experimentally investigated in an unsteady channel flow wherein the centerline velocity varies in time in a sinusoidal manner. The fluctuating wall shear stress and velocity signals are temporally filtered and subsequently phase averaged. It is shown that the outer structures corresponding to the low spectrum range have a constant time lag with respect to the centerline velocity modulation. The inner active structures, in particular those with a frequency band containing the mean ejection frequency of the corresponding steady flow dominate the dynamics of the near-wall unsteady turbulence. The structures respond to the imposed shear oscillations in a complex way, depending both on their characteristic scales and the thickness of the oscillating shear zone in which they are embedded.

Unsteady turbulent shear flows are encountered in many practical situations in aerohydrodynamics, aeroacoustics or biofluid dynamics. Past research on pulsed pipe or channel flows was first focused on the eventual effects of the forced velocity oscillations on the time-mean flow and second on the modulation characteristics of the oscillating velocity field and the near-wall turbulence. There is now an established consensus that:

- The time-mean flow is unaffected by the imposed unsteadiness even in the presence of large imposed amplitudes and frequencies that may cause reverse flow near the wall.

- In the imposed high-frequency regime, the oscillating shear is confined in the low buffer layer and leads to the coexistence of a purely oscillating viscous Stokes flow with an unaffected time-mean flow.

- The turbulence cannot follow the rapid imposed unsteadiness when the time period becomes comparable with the median time scale of the near-wall turbulence. The turbulent shear stresses become frozen during the oscillation cycle under these circumstances.

Despite significant advances in the understanding and modeling of forced internal wall flows, there is still some lack in understanding the reaction of the fine turbulence structure to imposed time periodical shear [1]. One of the questions that arise concerns the spectral characteristics of unsteady near-wall turbulence. We partly investigated these points in [2] by determining the impact of the unsteadiness on the inner and outer layer structures but only in the low buffer layer. We extend and discuss detailed results in this paper in the entire inner layer.

The experiments were performed in the unsteady water channel described in detail in [3]. The centerline velocity was held constant and equals $\bar{U}_{c}=17.5 \mathrm{~cm} / \mathrm{s}$. This corresponds to a friction velocity of $\bar{u}_{\tau}=0.85 \mathrm{~cm} / \mathrm{s}$ and a Reynolds number based on the half-height of the channel of $\operatorname{Re}_{h}=\bar{U}_{c} h / \nu=8800$. The imposed amplitude was $20 \%$ of the centerline velocity throughout the whole study. The imposed frequency in wall units $f^{+}=f\left(\nu / \bar{u}_{\tau}^{2}\right)$, where $\nu$ is the cinematic viscosity, varied by a factor of 24 from $f^{+}=2 \times 10^{-4}$ to $f^{+}=60 \times 10^{-4}$. Hereafter, ()$^{+}$will designate variables normalized by the viscosity and time mean shear velocity. The imposed frequency range investigated here covers $l_{s}^{+}=38-7$ in terms of the frequency parameter $l_{s}^{+}=\sqrt{1 / \pi f^{+}}$, which is the viscous Stokes length normalized by $l_{\nu}=\nu / \bar{u}_{\tau}$. The wall shear stress and the velocity measurements were performed by means of a flush-mounted TSI- $1268 \mathrm{~W}$ hot film at the wall and a TSI 1276-10 W hot film located in the flow. Further details are provided in Tardu and Vezin [2].

The classical triple decomposition is used. A quantity $q$ is decomposed into a mean $\bar{q}$, an oscillating $\widetilde{q}$, and fluctuating $q^{\prime}$ component. The angle brackets designate the phase average, i.e., $\langle q\rangle$ $=\bar{q}+\tilde{q}$. The modulation characteristics of $\langle q\rangle$ are described by the amplitude $A_{\tilde{q}}$ and phase $\phi_{\tilde{q}}$ of the fundamental mode. The relative amplitude $a_{\tilde{q}}=A_{\tilde{q}} / \bar{q}$ is also introduced for convenience.

In a way similar to Naguib and Wark [4], we use here three digital zero-phase shift filters; namely, Filter 0 with bandpass in wall units $\Delta f_{0}^{+}=0-0.0045$, Filter 1 (bandpass $\Delta f_{1}^{+}$ $=0.0055-0.022)$, and Filter $2\left(\Delta f_{1}^{+}=0.0316-0.0482\right)$ to identify outer (Filter 0$)$ and inner (Filters 1 and 2) structures and their characteristics. The filtering is processed through well-designed zero-phase shift 128-point finite impulse response digital filters. The reader is referred to [2] for further important details and related discussions.

The phase shifts of the filtered signals are denoted by $\Delta \phi_{i}$ $=\phi_{i u^{\prime} \tilde{u}^{\prime}}-\phi_{\widetilde{u}}$ with $i=0,1$, and 2 and the corresponding time lags by $\Delta t_{i}=\Delta \phi_{i} / 2 \pi f^{+}$. The time lag of the modulation of the contribution of the outer structures is remarkably constant in the viscous layer at $y^{+}<50$ with $\Delta t_{0, u^{\prime} \tilde{u}^{\prime}}^{+} \approx-75$ as shown in Fig. 1 . In the low loglayer, however, the behavior of $\Delta t_{0, u^{\prime} \tilde{u}^{\prime}}^{+}$changes appreciably. The phase shift $\phi_{0, u^{\prime} \tilde{u}^{\prime}}-\phi_{\tilde{u}}$ at $y^{+}=100$ decreases first sharply in the imposed low-frequency range $f^{+}<0.002$, becomes subsequently constant and joins the line $\Delta t_{0, u^{\prime} \tilde{u}^{\prime}}^{+} \approx-75$ only in the imposed high-frequency regime. Thus, the response time of the outer structures is constant in the viscous layer $y^{+}<50$ with a repercussion at the wall of $\Delta t_{0, \tau^{\prime} \tau^{\prime}}^{+} \approx-125$ independent of the imposed frequency. The time-lag difference $\Delta t_{0, \tau^{\prime} \tilde{\tau}^{\prime}}^{+}-\Delta t_{0, u^{\prime} \widetilde{u}^{\prime}}^{+} \approx-50$ may be expressed as $\Delta t^{+}=-y_{0}^{+} / v_{0}^{+}$where $y_{0}$ and $v_{c}$ stand, respectively, for the distance to the wall of the outer edge of the viscous layer and a characteristic wall normal velocity. One finds $\Delta t^{+}=-50$ by taking $y_{0}^{+}=50$ and $v_{c}^{+}=\left[\sqrt{\overline{v^{\prime} v^{\prime}}} / \bar{u}_{\tau}\right]_{y^{+}=y_{0}^{+}} \approx 1$, i.e., by assuming that the convection velocity is approximately equal to the rms wall normal velocity at the outer edge of the viscous layer. An equivalent assumption could be that the wall normal convection velocity in the viscous layer is about $v_{c}=\bar{u}_{\tau}$, as suggested by Eckelmann [5]. The constancy of $\Delta t_{0, u^{\prime} \widetilde{u}^{\prime}}^{+}$at $y^{+}<50$ points to the difference of the diffusion mechanism governing the passive eddies and the $\left\langle u^{\prime} u^{\prime}\right\rangle$ modulation. The lack of the diffusion of the outer eddies in the sense we discussed before is in concordance with the idealized inviscid picture of the passive structures.

Figures 2(a) and 3 show how the phase shift of the turbulent longitudinal intensity modulation related to the active eddies differs from the passive ones in the entire inner layer. At a given $y^{+}$, the phase shift $\phi_{1, u^{\prime} \tilde{u}^{\prime}}-\phi_{\widetilde{u}}$ decreases linearly until a critical imposed frequency $f_{c r}^{+}$beyond which $\phi_{1, u^{\prime} \tilde{u}^{\prime}}-\phi_{\widetilde{u}}$ is constant, or equivalently the time lag $\Delta t_{1, u^{\prime} \widetilde{u}^{\prime}}^{+}=\left(\phi_{1, u^{\prime} \tilde{u}^{\prime}}-\phi_{\widetilde{u}}\right) / 2 \pi f^{+}$decreases with $f^{+}$through $\Delta t_{1, u^{\prime} \tilde{u}^{\prime}}^{+} \propto 1 / f^{+}$. This is clearly perceptible in Fig. 2(a). The critical frequency depends upon $y^{+}$. The best physical 


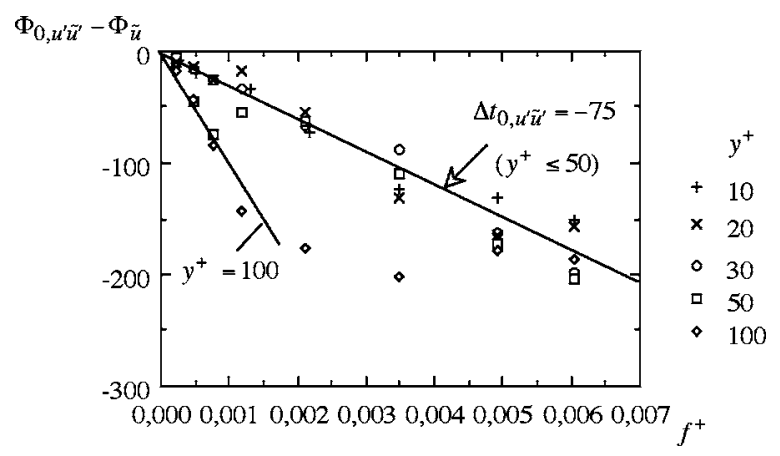

Fig. 1 Phase shift and corresponding time lag of the modulation of the longitudinal turbulent intensity depicted by Filter 0 at several wall normal positions in the inner layer

way to scale it is to use the Stokes length $l_{s}$ and relate the phenomena to the oscillating shear layer whose thickness is $2 l_{s}$. Figure $2(b)$ shows the distribution of $y_{s c r}^{+}=y^{+} / l_{s c r}^{+}$versus $y^{+}$. It is seen that $y_{s c r}^{+} \approx 2.5$, which is only slightly larger than the oscillating shear thickness, except in the low buffer region. Consequently, the response time of the structures 1 is constant in the oscillating shear layer and decreases in the plug flow zone wherein $\partial \widetilde{u} / \partial y \approx 0$.

The phase shift of the active structures 2 is small and remarkably independent of the imposed frequency in the viscous layer $y^{+}<50$ (Fig. 3). In the high logarithmic layer $\phi_{2, u^{\prime} \tilde{u}^{\prime}}-\phi_{\widetilde{u}}$ is closely similar to $\phi_{1, u^{\prime} \tilde{u}^{\prime}}-\phi_{\tilde{u}}$ of the structures 1 .

The relative amplitudes scaled with local $a_{\tilde{u}}$ are shown in Fig. 4

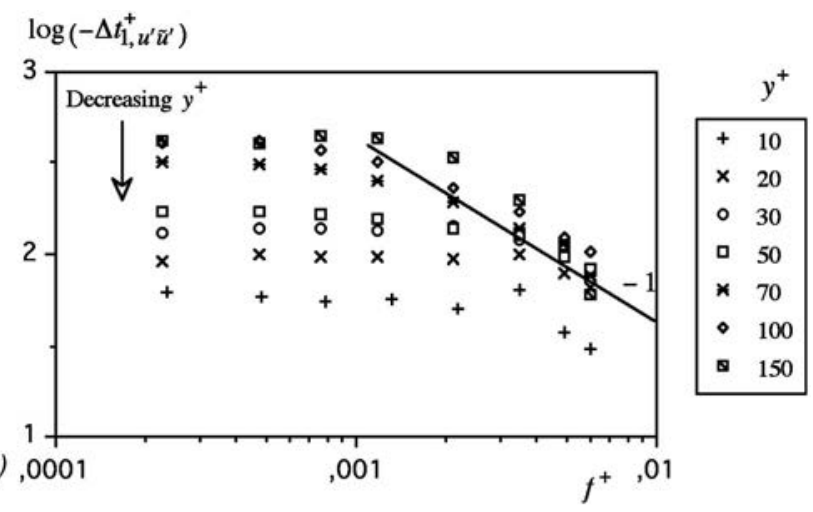

(b)

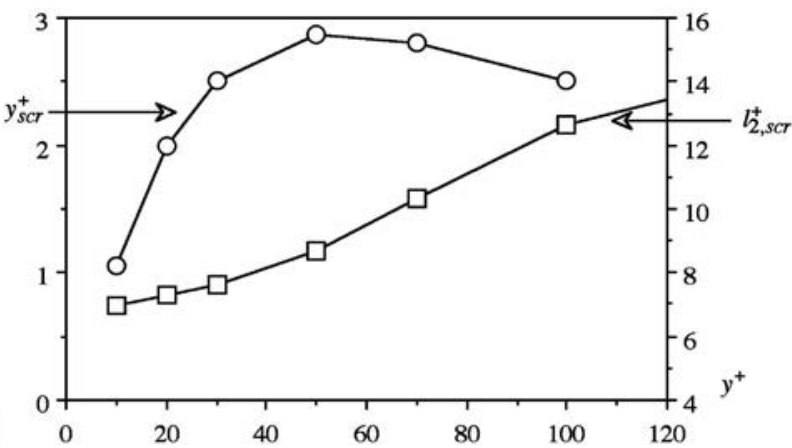

Fig. 2 Modulation characteristics of the inner structures depicted by Filter 1. (a) Time lag, (b) critical nondimensional frequency and wall normal distance beyond which the phase shift is constant (left) together with the critical Stokes length (right) related to the inner structures 2 (see the text).

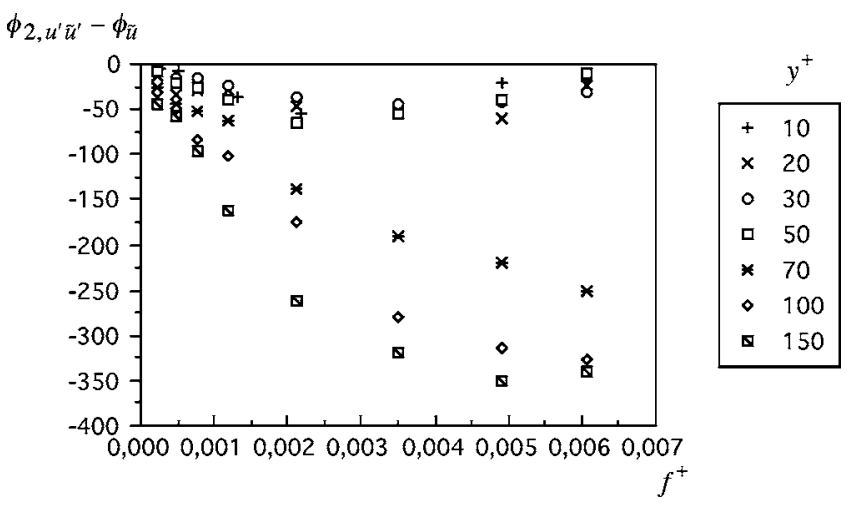

Fig. 3 Phase shift of the inner structures depicted by Filter 2 versus the frequency at different wall normal locations in the inner layer

versus the imposed frequency at four different $y^{+}$positions. The first striking observation emerging from these results is the sharp decrease of $a_{2, u^{\prime} \tilde{u}^{\prime}} / 2 a_{\tilde{u}}$ from large values of about 1 in the lowfrequency regime. The decrease is roughly linear from the quasisteady limit to some $f_{2, c r}^{+}$as shown by broken lines in Fig. 4 . The resulting critical Stokes lengths $l_{2, s c r}^{+}=\sqrt{\pi f_{2, c r}^{+}}$are reported in Fig. $2(b)$ at the right. It is seen that $l_{2, s c r}^{+}$increases in the buffer layer until it reaches a plateau region in the log layer. The relative amplitude of the large scale $a_{0, u^{\prime} \tilde{u}^{\prime}}$ and inner structures $a_{1, u^{\prime} \tilde{u}^{\prime}}$ do not significantly differ from the global response $a_{u^{\prime} \tilde{u}^{\prime}}$ in the entire inner layer.

The response of the inner eddies are recapitulated in Fig. 5. The active eddies 2 are closely related to the quasi-streamwise vortices (QSV), which are the major coherent structures in the buffer layer. They contribute mostly to the low- speed streak formation and Reynolds shear stress in steady [4] and unsteady flows [2]. The peculiar behavior of the cutoff in the response of the active structures 2 to imposed unsteadiness can tentatively be explained by the reaction of the QSV to the oscillating shear $\partial \tilde{u} / \partial y$. The QSV are entirely embedded in the oscillating shear zone when $y^{+}$ $=y_{s}^{+} l_{s}^{+} \approx 30$, which correspond to the top of the structures. Since $\partial \widetilde{u} / \partial y$ is constrained into $y_{s}^{+}<2$, this condition implies a critical Stokes length of $l_{2, s c r}^{+} \approx 15$, which corresponds well to the asymptotic limit in Fig. 2(b). The majority of the QSV's are in contact with the oscillating shear under this condition and the $\left\langle u^{\prime} u^{\prime}\right\rangle_{2}$ modulation extends to the log-layer. The active structures response is fast with small phase shifts $\phi_{2, u^{\prime} \tilde{u}^{\prime}}-\phi_{\widetilde{u}}$ in this zone $\left(y^{+}<50\right)$ and $\left\langle u^{\prime} u^{\prime}\right\rangle_{2}$ diffuses away resulting in larger time lags (Fig. 3). Only smaller structures in their initial stage of development are directly affected by $\partial \tilde{u} / \partial y$ when the latter is constrained into $y^{+}<15$ as it is shown at left in Fig. 5. Thus, the oscillating shear has to be concentrated sufficiently close to the wall, to stimulate the unsteady reaction of these merely immature QSV and to activate the $\left\langle u^{\prime} u^{\prime}\right\rangle_{2}$ modulation in the low buffer layer. This explains the occurrence of a minimum at $l_{2, s c r}^{+} \approx 7$ in Fig. $2(b)$.

The active structures 1 are larger scale eddies that extend beyond the buffer layer. Their time lag is constant in the oscillating shear zone and decreases in the plug layer. Figure 2 suggests that their size may reach $80-100$ wall units. The inactive eddies are large-scale motions associated mainly with pressure-strain correlations and turbulent diffusion according to Bradshaw [6]. They do not contribute to the shear stress production. They consequently scale with integral variables in steady flow contrarily to the active eddies scaling with the inner wall variables. The outer 

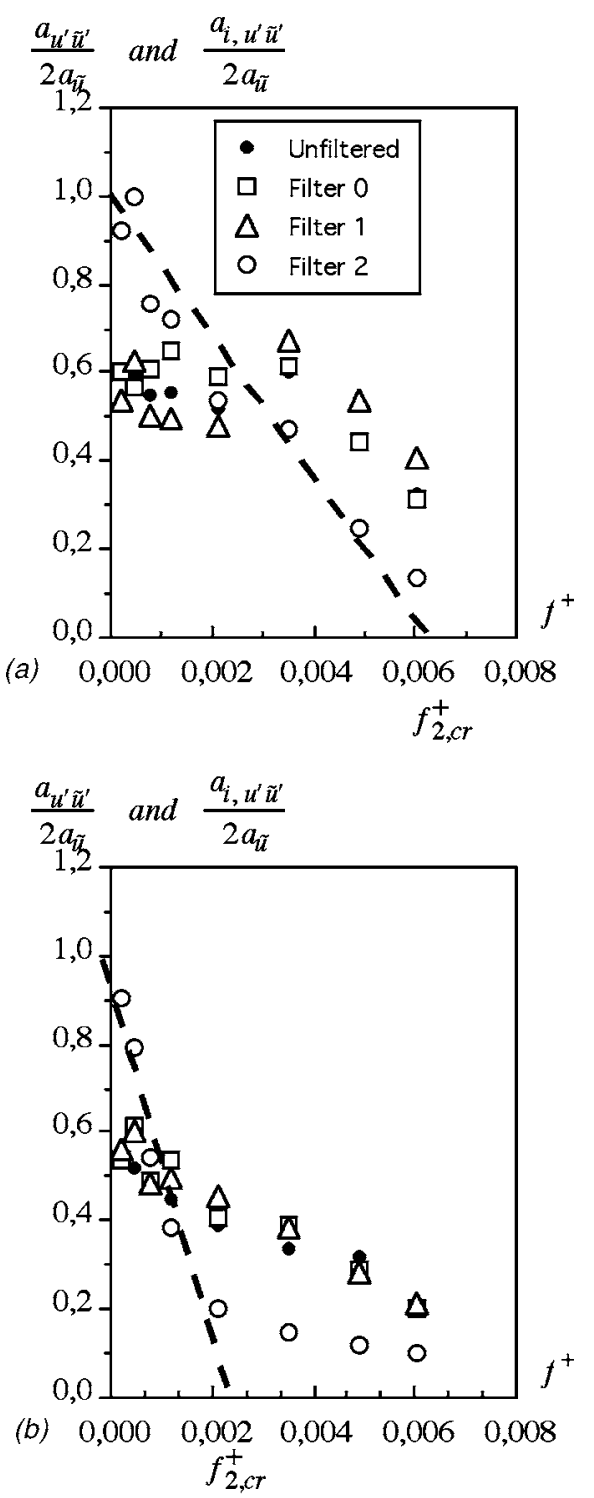

Fig. 4 Relative amplitude of the filtered and unfiltered streamwise fluctuating velocity at $(a) y^{+}=20,(b) y^{+}=100$

velocity scale in unsteady flows is the centerline velocity oscillations $\left\langle U_{C}\right\rangle$ and the inner velocity scale is the shear velocity modulation $\left\langle u_{\tau}\right\rangle$. Thus, outer and inner scaling would respectively imply $a_{u^{\prime} \tilde{u}^{\prime}} \approx 2 a_{u \tilde{c}}, \quad \phi_{u^{\prime} \tilde{u}^{\prime}} \approx \phi_{u \widetilde{c}}$ for the passive structures and $a_{u^{\prime} \tilde{u}^{\prime}}$ $\approx 2 a_{u \tilde{\tau}}=a_{\tilde{\tau}}, \phi_{u^{\prime} u^{\prime}}=\phi_{\tilde{\tau}}$ for the inner ones. Neither the amplitudes $a_{i, u^{\prime} u^{\prime}}$ nor the phases $\phi_{i, u^{\prime} u^{\prime}}$ obey these relationships except in the quasi-steady regime. The structures respond to the imposed shear oscillations in a complex way, depending both on their characteristic scales and on the thickness of the oscillating shear zone in which they are embedded.

The results presented here may be useful in the development of multiple-scale modeling in unsteady flows [7]. Consider to this end the modulation of the kinetic energy equation

$$
\frac{\partial \tilde{k}}{\partial t}=\tilde{P}-\widetilde{\varepsilon}-\frac{\partial}{\partial y}\left[\frac{1}{\rho} v^{\prime} \tilde{p}^{\prime}+v^{\prime} \tilde{k}-\nu \frac{\partial \tilde{k}}{\partial y}\right]
$$

where $\widetilde{P}$ and $\widetilde{\varepsilon}$ are, respectively, the production and dissipation, the term under the bracket stands for the turbulent and viscous diffusion, and where the second-harmonic production is neglected. We may write $\tilde{k}=\tilde{k}_{\text {Outer }}+\tilde{k}_{\text {Inner }}$ with $\tilde{k}_{\text {Outer }}=\tilde{k}_{0}, \tilde{k}_{\text {Inner }}=\widetilde{k}_{1}+\widetilde{k}_{2}$ since

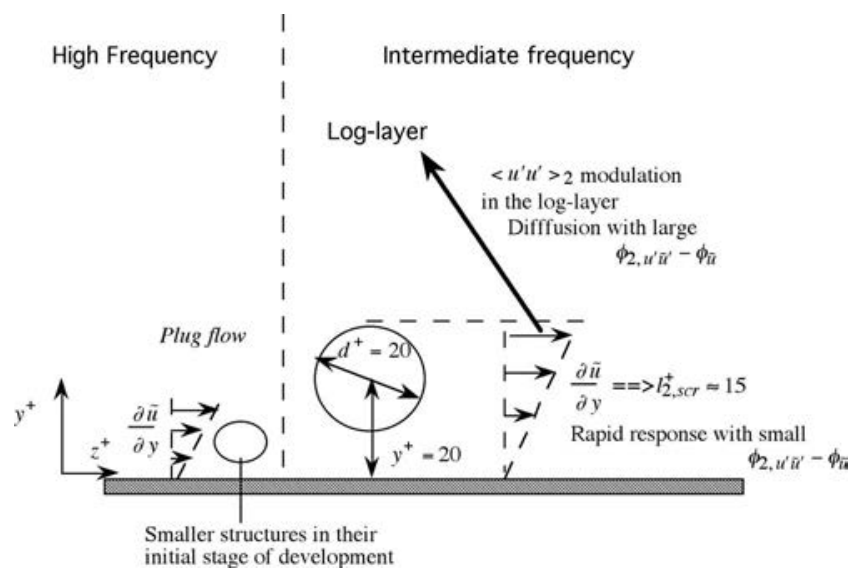

Fig. 5 Schematic representation of the frequency response of structures 2

these components fall into nonoverlapping parts of the spectrum. According to the results presented in this paper and the related discussion we made in the previous sections, the outer passive eddies contribute mainly to the turbulent diffusion. Thus,

$$
\frac{\partial \tilde{k}_{0}}{\partial t}=-\frac{\partial}{\partial y}\left[\frac{1}{\rho} v^{\prime} \tilde{p}^{\prime}+v^{\prime} \tilde{k}\right]
$$

The classical multi-scale cascade equations are written as

$$
\begin{gathered}
\frac{\partial \tilde{k}_{1}}{\partial t}=\widetilde{P}_{1}-\widetilde{\varepsilon}_{1}+\nu \frac{\partial^{2} \tilde{k}_{1}}{\partial y^{2}} \\
\frac{\partial \tilde{k}_{2}}{\partial t}=\widetilde{P}_{2}-\widetilde{\varepsilon}_{2}+\varepsilon_{1}+\nu \frac{\partial^{2} \widetilde{k}_{2}}{\partial y^{2}}-\frac{\partial \tilde{k}_{0}}{\partial t}
\end{gathered}
$$

where the coupling between the inner structures 1 and 2 takes place through the dissipation and the passive eddies intervene in the turbulent diffusion. The idea here is to couple these equations with the relationships based on the rapid distortion model in a way similar to Mankbadi and Liu [8] and Tardu and Da Costa [1] wherein substantial details can be found. The closure in these models is based on the effective strain parameter $\left\langle\alpha_{\text {eff }}\right\rangle$. The structural parameters such as the ratio of the Reynolds shear stress to the kinetic energy are related to $\left\langle\alpha_{\text {eff }}\right\rangle$ by $-\left\langle u^{\prime} v^{\prime}\right\rangle /\langle k\rangle=F\left(\left\langle\alpha_{\text {eff }}\right\rangle\right)$, where the function $F$ is obtained by the bench data of the steady turbulent flow. The transport equation for $\left\langle\alpha_{\text {eff }}\right\rangle$ in its simplest form is

$$
\frac{\partial\left\langle\alpha_{e f f}\right\rangle}{\partial t}=-\frac{\left\langle\alpha_{e f f}\right\rangle}{T_{d}(y)}+\frac{\partial\langle u\rangle}{\partial y}
$$

where $T_{d}(y)$ is the rapid distortion time scale. We propose here to combine Eqs. (3) and (4) with each individual transport equation for the effective strain parameters, i.e.,

$$
\frac{\partial\left\langle\alpha_{e f f}\right\rangle_{i}}{\partial t}=-\frac{\left\langle\alpha_{e f f}\right\rangle_{i}}{T_{d i}(y)}+\frac{\partial\langle u\rangle}{\partial y}
$$

where $i=0-2$ stands for the outer (0) and inner structures ( 1 and 2) and $T_{d i}(y)$ are the corresponding rapid distortion time scales. The latter can easily be estimated from the time lags $\Delta t_{i, u^{\prime} \tilde{u}^{\prime}}^{+}$investigated in this study. It is believed that this strategy can lead to more efficient modeling of the complex near-wall turbulent unsteady flows.

\section{References}

[1] Tardu, S.-F., and Da Costa, P., 2004, "Experiments and Modeling of an Unsteady Turbulent Channel Flow," AIAA J., 43(1), pp. 140-149. 
[2] Tardu, S.-F., and Vezin, P., 2004, "Response of the Streaks, Active, and Passive Eddies in an Unsteady Turbulent Channel Flow," Int. J. Heat Fluid Flow, 25(6), pp. 925-932.

[3] Tardu, S.-F., Binder, G., and Blackwelder, R.-F., 1994, "Turbulent Channel Flow With Large Amplitude Velocity Oscillations," J. Fluid Mech., 267, pp. 109-151.

[4] Naguib, A.-M., and Wark, C.-E., 1992, "An Investigation of Wall-Layer Dynamics Using a Combined Temporal Filtering and Correlation Technique," J. Fluid Mech., 243, pp. 541-560.

[5] Eckelmann, H., 1974, "The Structure of the Viscous Sublayer and the Adjacent
Wall Region in a Turbulent Channel Flow,” J. Fluid Mech., 65, pp. 439-459.

[6] Bradshaw, P., 1967, "Inactive Motion and Pressure Fluctuations in Turbulen Boundary Layers," J. Fluid Mech., 30, pp. 241-258.

[7] Launder, B.-E., 1984, "Second-Moment Closure: Methodology and Practice,' in Turbulence Models and Their Applications, Editions Eyrolles, Paris, pp. $1-143$.

[8] Mankbadi, R.-R., and Liu, J.-T.-L., 1992, "Near Wall Response in Turbulent Shear Flows Subjected to Imposed Unsteadiness," J. Fluid Mech., 238, pp. $55-71$. 